\title{
Erratum to: A Recount of Sunspot Groups on Staudach's Drawings
}

\section{Leif Svalgaard ${ }^{1}$}

Published online: 20 December 2016

(C) Springer Science+Business Media Dordrecht 2016

Erratum to: Solar Phys (2017) 292:4

DOI 10.1007/s11207-016-1023-x

We regret that on page 2, the phrase "Wolf using his small, but superb, 21.5-foot Fraunhofer refractor" should read "Wolf using his small, but superb, 2.5-foot Fraunhofer refractor".

Sunspot Number Recalibration

Guest Editors: F. Clette, E.W. Cliver, L. Lefèvre, J.M. Vaquero, and L. Svalgaard

The online version of the original article can be found under doi:10.1007/s11207-016-1023-x.

L. Svalgaard leif@leif.org

1 Stanford University, Cypress Hall C3, W.W. Hansen Experimental Physics Laboratory, Stanford University, Stanford, CA 94305, USA 